\title{
Pharmacokinetic considerations for pediatric patients receiving analgesia in the intensive care unit; targeting postoperative, ECMO and hypothermia patients
}

Gerdien A. Zeilmaker, Paula Pokorna, Paola Mian, Enno D. Wildschut, Catherijne A. J. Knibbe, Elke H.J. Krekels, Karel Allegaert \& Dick Tibboel

To cite this article: Gerdien A. Zeilmaker, Paula Pokorna, Paola Mian, Enno D. Wildschut, Catherijne A. J. Knibbe, Elke H.J. Krekels, Karel Allegaert \& Dick Tibboel (2018): Pharmacokinetic considerations for pediatric patients receiving analgesia in the intensive care unit; targeting postoperative, ECMO and hypothermia patients, Expert Opinion on Drug Metabolism \& Toxicology, DOI: $10.1080 / 17425255.2018 .1461836$

To link to this article: https://doi.org/10.1080/17425255.2018.1461836

Accepted author version posted online: 06

Apr 2018.

Published online: 11 Apr 2018.

Submit your article to this journal ¿

Џ Article views: 3

View related articles

View Crossmark data \lceil 


\title{
Pharmacokinetic considerations for pediatric patients receiving analgesia in the intensive care unit; targeting postoperative, ECMO and hypothermia patients
}

\author{
Gerdien A. Zeilmaker ${ }^{a *}$, Paula Pokorna ${ }^{\mathrm{a}, \mathrm{b}, c *}$, Paola Mian ${ }^{\mathrm{a}}$, Enno D. Wildschuta*, Catherijne A. J. Knibbe ${ }^{\mathrm{d}, e *}$, Elke H. \\ J. Krekels ${ }^{\mathrm{d*}}$, Karel Allegaert ${ }^{\mathrm{a}, \mathrm{f*}}$ and Dick Tibboel ${ }^{\mathrm{*} *}$ \\ antensive Care and Department of Pediatric Surgery, Erasmus MC-Sophia Children's Hospital, Rotterdam, The Netherlands; ${ }^{b}$ Department of \\ Pediatrics, General Faculty Hospital Prague, First Faculty of Medicine, Charles University and General University Hospital in Prague, Prague, Czech \\ Republic; Institute of Pharmacology, First Faculty of Medicine, Charles University and General University Hospital in Prague, Prague, Czech \\ Republic; 'Division of Pharmacology, LACDR, Leiden University, Leiden, The Netherlands; eDepartment of Clinical Pharmacy, St. Antonius Hospital, \\ Nieuwegein, The Netherlands; fDepartment of Development and Regeneration, KU Leuven, Leuven, Belgium
}

ABSTRACT

Introduction: Adequate postoperative analgesia in pediatric patients in the intensive care unit (ICU) matters, since untreated pain is associated with negative outcomes. Compared to routine postoperative patients, children undergoing hypothermia (HT) or extracorporeal membrane oxygenation (ECMO), or recovering after cardiac surgery likely display non-maturational differences in pharmacokinetics (PK) and pharmacodynamics (PD). These differences warrant additional dosing recommendations to optimize pain treatment.

Areas covered: Specific populations within the ICU will be discussed with respect to expected variations in PK and PD for various analgesics. We hereby move beyond maturational changes and focus on why PK/PD may be different in children undergoing HT, ECMO or cardiac surgery. We provide a stepwise manner to develop PK-based dosing regimens using population PK approaches in these populations.

Expert opinion: A one-dose to size-fits-all for analgesia is suboptimal, but for several commonly used analgesics the impact of $\mathrm{HT}$, ECMO or cardiac surgery on average PK parameters in children is not yet sufficiently known. Parameters considering both maturational and non-maturational covariates are important to develop population PK-based dosing advices as part of a strategy to optimize pain treatment.
ARTICLE HISTORY

Received 22 April 2017

Accepted 3 April 2018

\section{KEYWORDS}

Pediatric; intensive care; post-operative; hypothermia; ECMO; cardiac surgery; pharmacokinetics; pharmacodynamics; population PK

\section{Introduction}

The importance of post-operative pain relief in neonates and infants became apparent after the landmark publication by Anand and Aynsley-Green [1]. While untreated pain results in prolonged pediatric intensive care unit (ICU) stay and increases the children's stress responses [2,3], overtreatment may result in prolonged artificial ventilation. Repetitive painful stimuli may induce hypersensitivity to pain and negative behavioral consequences in later life; longitudinal data on this issue in humans are limited [4-7].

The World Health Organization (WHO) has published guidelines on the pharmacological treatment of pain in children (2012) $[8,9]$. These general guidelines are also often applied to children admitted to specialized PICUs. Currently, approximately $50 \%$ of the current patient case mix in many PICUs concerns post-operative patients of various surgical specialties, including those admitted for surveillance of vital functions and/or undergoing hypothermia. Post-operative pain is hereby defined as pain within the first $48 \mathrm{~h}$ after surgery. Standard systemic pharmacotherapy for mild postoperative pain consists of acetaminophen (paracetamol) and non-steroidal anti-inflammatory drugs (NSAIDS). For moderate and severe pain, opioids are recommended as part of multimodal analgesia [10-12]. Although these WHO guidelines provide a framework with specific emphasis on the two stage, multimodal approach (non-opioids to opioids) and on dosing suggestions for different analgesics, they still fail to catch the full spectrum of variability and heterogeneity, sticking to the concept of a one-dose to size or age-fits-all for analgesia [8,9]. Particularly, children undergoing hypothermia (HT); extracorporeal membrane oxygenation (ECMO) or after cardiac surgery) may benefit from fine tuning that takes into account the pharmacokinetics (PK), pharmacodynamics (PD), but also pharmacogenetics (PG) and disease characteristics (SIRS,HT, ECMO or cardiac surgery or renal impairment) to reach an optimal dosing regimen for post-operative pain relief. PK parameters such as volume of distribution (Vd) and clearance $(\mathrm{CL})$ are expected to differ in maturational (e.g. size, weight, age) and non-maturational (HT, ECMO, cardiac surgery, disease severity, including renal impairment) variables. Validated PD assessment tools have been developed to assess the clinical effects of pain treatment $[13,14]$, but incorporating the end points in a dosing model remains challenging, while the assessment results may also be affected by the disease characteristics. While knowledge on PG is available for several genetic polymorphisms

CONTACT D. Tibboel d.tibboel@erasmusmc.nl E Intensive Care and Department of Pediatric Surgery, Erasmus MC-Sophia Children's Hospital, Room SK-3284, Wytemaweg 803015 CN, Rotterdam, The Netherlands

"These authors contributed equally to this work

(c) 2018 Informa UK Limited, trading as Taylor \& Francis Group 


\section{Article highlights}

- PK-based dosing regimens are lacking for routinely used analgesics in specific groups of (preterm) neonates, infants or children in specific subgroups, like e.g. HT, ECMO, or cardiac surgery.

- PK parameters depend not only on maturational, but also on nonmaturational changes. These covariates, combined with intra-patient variability throughout the disease process further add to the variability in PK/PD of analgesics and the choice of the drug throughout childhood.

- PD assessment is necessary but difficult given the diversity of the patient population and their disease characteristics. Its variability further adds to the intra- and interpatient variability observed in the PK/PD relationship.

- New techniques in modelling, such as (semi-)physiologic models and PK/PD-based models, may better describe the clinical situation and estimate the extent of differences between specific subpopulations.

This box summarizes key points contained in the article

involved in drug PK or PD, it is not yet routinely applied in clinical practice $[15,16]$.

Critically ill pediatric patients tend to display a large variability in PK and PD compared to the average pediatric ICU population or compared to patients after less extensive surgery. To illustrate this, due to mechanisms underlying cold-induced pain, the pain expression of children undergoing $\mathrm{HT}$ setting differs from that in children not exposed to HT (different cry, facial response, no shivering, no muscles tremor and decreased muscle contractions) $[17,18]$. Several studies have recently been undertaken to provide insight in the changes in PK in these specific patient groups $[19,20]$. Similarly, ECMO or cardiac surgery likely affects the PK (larger Vd, decreased CL) of several analgesics.

In this review, we aim to integrate both maturational (e.g. size, weight, age) and non-maturational characteristics that affect intraand inter-patient variability in PK/PD, and to identify patient groups that are expected to differ in PK and PD compared to the general pediatric ICU population. We will first describe the general aspects of PK, focusing on drug absorption, distribution, metabolism, and excretion (ADME) and PD of analgesics in neonates, infants and children [21]. We will then focus on the earlier mentioned non-maturational characteristics and the expected variation in PK and PD. The magnitudes and effects of specific patient and disease characteristics on PK and PD parameters are described. However, a direction or magnitude of effect cannot always be given, since the direction may go both ways depending on covariates. Also the magnitude may vary depending on the patient and clinical circumstances. Subsequently we explore the consequences of these variations, working toward a PK and PD based dosing recommendation for these specific patients. In the expert opinion section, we focus on tools for future research to close the current knowledge gap, including suggestions to integrate this knowledge in population PK/PD modeling.

\section{Maturational and non-maturational related changes}

\subsection{Pharmacokinetics}

In clinical pharmacology, drug-related effects are predicted from compound- and population-specific PK and PD. The general PK principles of drugs hereby apply, irrespective of population-specific characteristics. The absorption, distribution, metabolism, and elimination (ADME) of analgesic drugs in neonates, young infants and children are illustrated in Table 1. Absorption is described by the absorption rate constant $(\mathrm{Ka})$, the time to reach the maximum (peak) plasma concentration (Tmax) and drug bioavailability (F). F is the fraction of an administered dose of unchanged drug that reaches the systemic circulation and is typically $100 \%$ upon intravenous administration [22]. Absorption can be affected by maturational and non-maturational changes (Table 1). Distribution is represented by the $\mathrm{Vd}$ of a central compartment and in some cases by the Vd of one or more peripheral compartments that are in equilibrium with the central compartment. For example, a peripheral compartment that is used as PK sample site for the central nervous system is the cerebrospinal fluid (CSF), which is in close equilibrium with the neurons in the brain, where the effect of opioids are expected to occur [23]. More lipophilic opioids will diffuse faster across the blood-brain barrier - with faster onset of analgesia [24]. Also, distribution can be affected by maturational and non-maturational changes (Table 1). Metabolic or primary renal elimination is commonly expressed as clearance $(C L)$, while the elimination half-life $\left(t_{1 / 2}\right)$ represents the time it takes to reduce by half the drug concentration. By definition, in a one-compartment model, the elimination half-life is influenced by both $\mathrm{Vd}$ and $\mathrm{CL}$, since $\mathrm{CL}=\mathrm{k} \cdot \mathrm{Vd}$ with $\mathrm{t}_{1 / 2}=0.693 / \mathrm{k}$, with $\mathrm{k}=$ elimination rate constant) [25] .

Simple extrapolation of PK or PD estimates from adults to pediatric patients is obsolete. Both PK and PD processes change with a child's growth and development, but these factors are collinear. Growth relates to the increases in weight, length and size with proportional or disproportional changes in body proportions, body composition and organ weights, and associated changes in activity or function (e.g. barrier functions, renal clearance, or hepatic drug metabolism) as reflected in the maturational changes in the ADME patterns. This is predominantly observed in infancy, and most notably in prematurely born children [26]. Thus, within the pediatric-age range the inter-individual variability in PK and PD for almost all drugs is higher than that in adults $[27,28]$. Age, not weight, is key in the ontogeny of hepatic and intestinal drug transporters, with increases or decreases of specific transporters depending on age [29]. These transporters are important to both drug absorption and elimination of drugs. Even when we only focus on PK, variability between and within a specific population occurs up to the level of clinical relevance, as ignoring the variability may result in concentrations below or above the therapeutic range. This variability is explored with

Table 1. General aspects of ADME of analgesic drugs on PK in neonates, young infants and children.

\begin{tabular}{llll}
\hline PK covariates & \multicolumn{1}{c}{ Absorption } & \multicolumn{1}{c}{ Distribution } & $\begin{array}{c}\text { Elimination } \\
\text { (Metabolism/ } \\
\text { Excretion) }\end{array}$ \\
\hline Maturation & $\uparrow$ or $\downarrow$ & Variable or $\downarrow \uparrow$ & Variable or $\downarrow \uparrow$ \\
Asphyxia & $\downarrow$ or variable & Variable NS or $\downarrow \uparrow$ & Variable or $\downarrow \uparrow$ \\
Sepsis (SIRS) & No data & Variable or $\downarrow \uparrow$ & Variable or $\downarrow \uparrow$ \\
Hypothermia & Decreased $\downarrow$ & Decreased $\downarrow$ & Decreased $\downarrow$ \\
ECMO & No data & Increased $\uparrow$ & Decreased $\downarrow$ \\
\hline
\end{tabular}

NS: not significant. Updated and used with permission from P. Pokorna, 2015 [65]. 
the use of covariates, subdivided in maturational (size, weight, age) and non-maturational characteristics (disease and/or treatment dependent), together with PG characteristics (Figure 1). For these ADME-related processes, maturational covariates have been reported, as summarized below.

Absorption: Following oral administration, absorption displays extensive maturation because of gastro-enteral maturation (e.g. anatomy, motility, drug metabolism or transporters). Also non-enteral routes (e.g. cutaneous, muscular size, inhalation and circulation) display age-related changes. Distribution: Although a 'theoretical volume,' distribution volume depends on physical (e.g. extra- and intracellular water, lipophilic or water soluble compound, ionization and protein binding) and physiologic (protein binding, tissue uptake, permeation to deep compartments) processes. Consequently, the distribution volume is also driven by maturational changes and disease characteristics.

Metabolism: The drug metabolizing maturational activity of the different isoenzymes is enzyme specific and determined by age (postnatal age, postmenstrual age) or size. The expression and activity of cytochrome p450 (CYP) isoenzymes - proteins that catalyze phase I metabolism of many drugs - change dramatically from fetal life through adolescence. At birth, the overall CYP content and activity is $30-60 \%$ of adult activity when expressed per gram of liver [30]. With advancing age, CYP-related metabolism increases, but the different isoenzymes of the P450 family show different developmental patterns [30]. In addition to phase I metabolism, phase II metabolic pathways such as glucuronidation and sulfation also display maturational changes.

Excretion: the most relevant route is the renal route, both through glomerular filtration and renal tubular transport.
These processes do not mature simultaneously and also relate to both postnatal age and weight. We refer the reader to some recent reviews on the maturational aspects of drug disposition throughout childhood $s[26,31,32]$.

Obviously, these maturational changes have an important impact on drug disposition of analgesics, resulting in changes in PK parameters, as illustrated for acetaminophen, but this exercise can also be performed for opioids. Changes in acetaminophen absorption (gastric emptying) and Vd (body composition) have been quantified across neonates and adults [33]. In addition, CL increases with age and/or size [27,28,34]. The complex influence of these maturational covariates has also been illustrated for acetaminophen: Wang et al. documented that acetaminophen $\mathrm{CL}$ changes nonlinearly with bodyweight [33]. Interestingly, the authors provided dosing guidelines for the age range from preterm neonates to adolescents. resulting in similar exposure across these age range [33]. These changes in the clearance reflect both maturational changes in hepatic metabolism and subsequent renal elimination [35].

In addition to these maturational changes, non-maturational changes such as disease-dependent (asphyxia, sepsis, renal impairment, systemic inflammatory response syndrome (SIRS)) [36,37], and multiple organ dysfunction syndrome (MODS) or treatment modalities (HT, ECMO) [38] can further influence PK parameters, leading to additional variability between and within patients (Table 1 and Figure 1). Acute kidney injury in critically ill neonates receiving ECMO warrants dosage adjustment or even avoidance of nephrotoxic drugs [39]. At present, the numbers of evidence-based observations and estimates on the impact of these covariates are limited. Moreover, the distinction between maturational and non- (a)
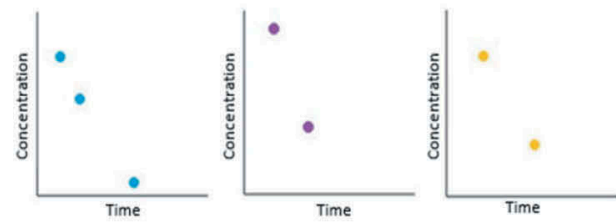

(b)

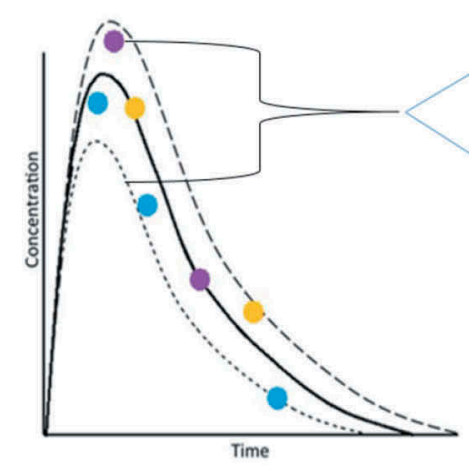

(c)

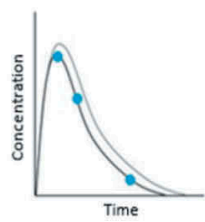

Figure 1. Concentration-time profiles of individual patients (blue, purple, yellow) (a), concentration-time profile of population; including the variability (residual errors, intra- and inter-individual variability) (b), individual profiles related to population concentration-time profile (c). Full color available online. 
maturational covariates cannot always be made, as in the case of genetic polymorphisms or renal impairment.

Genetic polymorphisms cannot simply be considered nonmaturation related changes. The impact of polymorphisms in e.g. cytochrome-P-450 (CYP) enzyme activity can change throughout the childhood age range(maturational), as has been documented for CYP2D6 or CYP2C19 [40]. Therefore, PG should be considered a potential covariate in individualized pain management in children of different ages. At present, dosing in clinical practice is not based on the information on PG differences amongst children explaining inter-patient variability is not yet taken into account for dosing in clinical practice $[15,41]$. However, it has been reported that PG can have an impact on outcome - including serious adverse reactions due to drug toxicity - $[42,43]$.

The frequency of genetic polymorphisms varies between racial and ethnic populations, with differences in allele functionality between European Caucasians, Asians and AfricanAmericans of all ages [16]. Polymorphisms in neonates receiving tramadol in a postoperative setting have been extensively described for CYP2D6 and organic cation transporter1 (OCT1). Implementation of a CYP2D6 activity score showed that inter-individual variability in metabolism of tramadol to the tramadol metabolite $\mathrm{CL}$ could partly be explained by CYP2D6 polymorphism [15,41]. Furthermore, polymorphism in OCT1 (reuptake transporter in the hepatocyte) has demonstrated that the ratio metabolite/tramadol is higher with $<2$ functional copies. Tramadol also illustrates the impact of maturational and non-maturational changes in renal elimination clearance. The plasma tramadol metabolite concentrations will not only depend on their formation, but also on its subsequent renal elimination. This means that metabolite accumulation is more likely to occur in early infancy (renal elimination matures slower than hepatic metabolism) or in the context of renal impairment [41]. A similar pattern has been described for morphine during HT, with accumulation of morphine metabolites [44].

Exploring the impact of covariates on the inter- and intraindividual variability in PK is relevant as these covariates can subsequently be used to develop PK-based dosing regimens for the subpopulations or even individual patients.

\subsection{Pharmacodynamics}

The clinical efficacy of any intervention should be established using validated PD endpoints [45]. As with PK, there is a large inter and intra-variability in PD, with different PD endpoints being used, while a clear correlation between plasma concentration and for instance pain scores is often lacking [46].

Although integrated PK/PD studies have been published, PK data are usually reported separately or without integration with validated PD endpoints. PKPD modeling incorporating PD end points in a model is difficult and currently PKPD modeling studies are scarce for children. Moreover, validated PD assessment tools are highly tailored to specific age groups and circumstances as reflected by a mission statement paper from the European Society for Pediatric and Neonatal Intensive Care (ESPNIC) [45]. For instance, the COMFORT-B scale is a validated post-operative pain instrument for children
0-3 years, including pre-verbal neonates and infants. It detects meaningful effects of pain treatment, but cannot be used during muscular relaxation [13,14]. The same holds true for the Face, Legs, Activity, Cry and Consolability (FLACC) scale. Another scale, the CRIES, consists of the following items: Crying; Requires increased oxygen administration; Increased vital signs; Expression; Sleeplessness, and was specifically developed for post-operative infants $[47,48]$.

Interestingly, the observed variability in PD can also in part be explained by polymorphisms. For polymorphisms are not limited to PK, but can also affect PD related outcome variables. This was illustrated for the link between combined opioid mureceptor (OPRM1, opioid receptor) and Catechol-0-methyltransferase (COMT, intracellular signaling) polymorphisms and the need for rescue morphine in mechanically ventilated newborns $[49,50]$. To date the role of PG is still limited, but should at least be considered when an abnormal drug response is observed following surgery.

\section{Considerations to treat postoperative pain in neonates, young infants, and children: from one dose to size-fits-all toward patient tailored treatment}

Pain treatment is warranted in case of operative procedures, for specific neonatal anomalies, underlying critical disease [51] and specific conditions [52]. Optimal postoperative analgesia decreases stress and improves recovery and clinical outcome [53]. However, associations between neuro-apoptosis, neurological outcome and exposure to analgesics have been observed in animal models, while the clinical implications for adults and children are still hard to interpret. The American Society of Anesthesiologists' 2012 Practice Guidelines for Acute Pain Management in the Perioperative Setting provided guidance on the prevention and treatment on pain. Designing a care plan tailored to the individual and the surgical procedure involved is recommended - with an emphasis on multimodal regimens and regular pain assessment [54]. In children within the ICU setting, this commonly translates to intravenous administration of opioids and non-opioid analgesics.

Acetaminophen, morphine and fentanyl are the most widely used analgesic drugs. Multimodal analgesia in postoperative children (average age 4 year), acetaminophen combined with morphine, resulted in an opioid sparing effect [55]. The PK data hereby resulted in the use of loading doses with patient-tailored maintenance dosages for IV morphine or acetaminophen [56], subsequently guided by validated assessment (PD) tools. This concept is strongly supported in the WHO guidelines on treatment of pain although the evidence for the use of multimodal analgesia in children is still limited (WHO guidelines 2012) [9]. For other analgesics, the available data are even more limited [57,58]. Tramadol [59], nalbuphine [60], and ketorolac [61] seem to be promising and safe alternative drugs for postoperative care, although pain relief was considered to be less effective for nalbuphine compared to other opioids. Sufentanil may be another alternative, but this has been hardly studied in children [62,63] and neonates [64]. The variability in PK of analgesics in neonates, young infants and children is further illustrated in Table 2. 
As discussed earlier, special conditions like HT, ECMO or cardiac surgery are likely to further affect PK, and likely also PD.

\subsection{Hypothermia}

Evidence-based guidelines on pain management for neonates and children who undergo HT are still lacking. However, analgesics (e.g. opioids, mainly morphine) are commonly combined with sedatives (e.g. midazolam) in term neonates exposed to HT after perinatal asphyxia $[65,66]$. Evidence based data on PK of drugs in neonates and children who underwent HT have been reviewed by van den Broek et al. 2010 [66] and Pokorna et al. 2015 [65], but only few data are available on PK of analgesics or on PK in cases when perinatal asphyxia indicated HT. PK changes in neonates and children who underwent HT are known in terms of reduction in absorption (due to low splanchnic flow) for non-parenteral administered drugs (for oral or rectal route of administered acetaminophen), changes in distribution (decreased $\mathrm{Vd}$ of lipophilic drugs) or in CL. The impact of HT on morphine (PK) in neonates undergoing HT on indication of perinatal asphyxia has been quantified as a $23 \%$ decrease in morphine $\mathrm{CL}$ [44].

The PHARMACOOL study illustrates how a pragmatic study design with opportunistic sampling can provide more insight in the effect of HT on the PK of frequently used drugs in these neonates. We therefore suggest to focus on short-acting and rapid-onset opioids (e.g. alfentanil, nalbuphine, remifentanil) or intermediate-acting drugs (tramadol) guided by validated PD scores. Once the PK aspects have been elucidated, we can explore PD-related aspects since HT also affects pain expression by mechanisms related to cold-induced pain $[17,18]$ while thermal stimuli are initiated via thermoreceptors and the nociception may be sensitized under HT [17]. In general, a surgical procedure will not be performed under hypothermia if the procedure is not considered lifesaving. The patient will be rewarmed first, undergo neurological evaluation, and surgery is considered once the neurological condition has stabilized.

\subsection{Extracorporeal membrane oxygenation}

ECMO provides temporary respiratory and cardiac support to critically ill neonates and children with Multiple Organ Dysfunction (MODS) if conventional treatment has failed. Optimal analgesia is essential during the ECMO run and during cannulation and decannulation [67]. Similar to HT, the effects of ECMO superimpose on the underlying disease and thereby further affect the maturational and non-maturational PK changes of analgesics [68,69]. Compound-specific drug losses in the ECMO circuit have been quantified in different in vitro studies $[68,70]$. PK of analgesic drugs is hereby also affected by drug-dependent characteristics (drug lipophilicity, protein binding capacity, and drug sequestration). In vivo, the final observed phenotypic disposition covers both maturational, disease-related and equipment-related aspects. For high lipophilic drugs like fentanyl or sufentanil [70], ECMO leads to an increased Vd and decreased CL. ECMO flow was found to have a small effect on M3G metabolite clearance. An increase in ECMO blood flow was associated with decreased clearance, possibly reflecting illness severity. Dopamine dose reflected decreased renal CL [71,72].

\subsection{Pain treatment after cardiac surgery}

Current guidelines on pain treatment after cardiac surgery in children are not yet based on population tailored PK or PD. The main guidelines on this issue were provided by the association of Paediatric Anaesthetists of Great Britain and Ireland in 2012 but are based on small, nonrandomized trials [73]. Morphine is recommended as the analgesic of first choice, but very little PK and PD data on this drug are presented in this guideline, despite the fact that PK and PD based dosing guidelines have been established for non-cardiac surgery in children [19]. Routinely used analgesics and sedatives in cardiac critical care are discussed in the recent consensus statement by Lucas et al. [74].

Several studies show differences in morphine serum concentration in children after cardiac surgery and children after non-cardiac surgery. Dagan et al. found that the use of

Table 2. Clinical studies on PK of selected analgesic drugs - opioids and NSAIDs (IV) in neonates, young infants and children.

\begin{tabular}{lll}
\hline Drug & \multicolumn{1}{c}{ Volume of distribution ${ }^{\mathrm{a}}$} & \multicolumn{1}{c}{ Clearance $^{\mathrm{a}}$} \\
\hline Alfentanil [112] & Neonates: $\mathrm{V}_{\text {dbeta }} 1 \mathrm{~L} / \mathrm{kg}$ & Neonates: $2.2 \pm 2.4 \mathrm{ml} / \mathrm{kg} / \mathrm{min}$ \\
& Children: $0.163-0.48 \mathrm{~L} / \mathrm{kg}$ & Infants and children: $5.9-11.1 \mathrm{ml} / \mathrm{kg} / \mathrm{min}$ \\
Ketorolac [55] & $4-8$ years: $0.19-0.44 \mathrm{~L} / \mathrm{kg}$ & $2-18$ months: \\
& & S-enantiomer $4.4-5 \mathrm{ml} / \mathrm{kg} / \mathrm{min}$ \\
& & R-enantiomer $1-1.04 \mathrm{ml} / \mathrm{kg} / \mathrm{min}$ \\
Morphine [19] & Vcentral: $46-81.2 \mathrm{~L} / 70 \mathrm{~kg}$ & $1.62 \mathrm{~L} / \mathrm{min} / 70 \mathrm{~kg}$ \\
& Vperipheral: $128 \mathrm{~L} / 70 \mathrm{~kg}$ & \\
Acetaminophen [27] & $0.179-17.2929 \mathrm{~L}(\mathrm{BW} 0.5-50 \mathrm{~kg})$ & $0.047-13.422 \mathrm{~L} / \mathrm{h}(\mathrm{BW} 0.5-50 \mathrm{~kg})$ \\
Sufentanil [57] & $2.9 \mathrm{~L} / \mathrm{kg}$ & $30.5(8.8) \mathrm{ml} / \mathrm{kg} / \mathrm{min}$ \\
Remifentanil & $0-2$ years: $0.452(0.144) \mathrm{L} / \mathrm{kg}$ & $0-2$ years: $90.5(36.8) \mathrm{ml} / \mathrm{kg} / \mathrm{min}$ \\
[77,78] & $2-6$ years: $0.240 \mathrm{~L} / \mathrm{kg}$ & adolescents: $57.2(21.2) \mathrm{ml} / \mathrm{kg} / \mathrm{min}$ \\
& $7-12$ years: $0.249 \mathrm{~L} / \mathrm{kg}$ & \\
Nalbuphine [54] & Vcentral: $210 \mathrm{~L} / 70 \mathrm{~kg}$ & $130 \mathrm{~L} / \mathrm{h} / 70 \mathrm{~kg}$, inter-compartment clearance $75.6 \mathrm{~L} / \mathrm{h} / 70 \mathrm{~kg}$ \\
& Vperipheral: $151 \mathrm{~L} / 70 \mathrm{~kg}$ & \\
Tramadol [36,53] & Neonates: fixed metabolite $224 \mathrm{~L} / 70 \mathrm{~kg}$ & $0-2$ years: $90 \mathrm{ml} / \mathrm{kg} / \mathrm{min}$ \\
& $0-2$ years: $0.2-0.3 \mathrm{~L} / \mathrm{kg}$ & $2-12$ years: $60 \mathrm{ml} / \mathrm{kg} / \mathrm{min}$
\end{tabular}

${ }^{\mathrm{a}}$ Mean (SD) PK parameters reported from different studies in these populations.

IV: intravenous; BW: body weight; L: liter; h: hour; min: minutes; kg: kilogram. 
inotropic support, e.g. epinephrine, dopamine or dobutamine, at more than $10 \mathrm{mcg} / \mathrm{kg} / \mathrm{min}$ after surgery significantly decreased morphine $C L$ from $1.5 \pm 0.41$ to $0.73 \pm 0.3 \mathrm{~L} / \mathrm{kg} / \mathrm{h}$. This is most likely due to changes in cardiovascular status, and a $50 \%$ reduction in morphine dosage was recommended for these children [75]. Lynn et al. also found decreased morphine $\mathrm{CL}$ in children after cardiac surgery as compared to agematched peers after non-cardiac surgery, but attributed this to slower maturation in the cardiac surgery patients [76]. In contrast, Elkomy et al. more recently were unable to find differences of morphine $C L$ in children after cardiac surgery and children after non-cardiac surgery [77]. Morphine serum levels seemed to be dependent on age and renal function, with renal function being often impaired after cardiac surgery [78]. Alternatively, Valkenburg et al. showed an increased Vd and a decreased morphine $C L$ in children after cardiac surgery compared to non-cardiac surgery [79].

PK parameters in children after cardiac surgery have been reported for remifentanil, clonidine, and dexmedetomidine. Two studies on remifentanil have been published in the last decade, both reporting maturational and non-maturational changes in children after cardiac surgery compared to noncardiac surgery [80,81]. Due to an 2.4 times increase in $\mathrm{Vd}$ during $C P B$ and post-CPB compared to pre-CPB values, a supplemental bolus dose of remifentanil of $25 \mu \mathrm{g}$ is proposed at the start of CPB to achieve the target concentration of $14 \mathrm{ng} / \mathrm{ml}$ in $50 \%$ of patients [81]. While clonidine was initially used as a sedative, it is currently also used as an analgesic for its favorable hemodynamic profile and mild analgesic effects $[82,83]$. For intravenous clonidine administration, the $\mathrm{Vd}$ (central volume $123 \%$, peripheral volume $126 \%$ ) but not $C L$, was increased in neonates and children after cardiac surgery [84]. The dosing recommendation for clonidine was only driven by maturational changes in neonates and infants, unrelated to cardiac surgery., Su et al. recently published a PK-based dosing advice for dexmedetomidine in neonates and children after cardiac surgery [85]. Both maturational (weight and age), and non-maturational (presence of intra-cardiac shunt and total bypass time) affected CL. Due to decreased $C L$ during the first two weeks of life, a $30-40 \%$ dose reduction is required in neonates to achieve similar steady state plasma concentration when compared to infants. Alternatively, $\mathrm{CL}$ is increased by $24 \%$ in the presence of a right-to-left intra-cardiac shunt.

The PK in children after cardiac surgery can be expected to differ from that in children after non-cardiac surgery. This can be mainly ascribed to the perioperative use of CPB, which has profound effects on the PK because of (1) hemodynamic changes, (2) hemodilution, (3) HT, (4) SIRS, (5) sequestration of drugs in the CPB system. These effects change constantly during $C P B$ and continue to have effects after weaning from CPB $[78,86]$.

(1) Hemodynamic changes during CPB are due to a decrease in blood pressure and the related changes in blood flow. These changes can arise because of nonpulsatile flow, a decrease in peripheral vascular resistance and the use of inotropes in hemodynamically unstable patients $[75,87]$.
(2) At the onset of CPB, priming fluid dilutes the patient's blood, to sometimes doubling of the circulating volume. Due to low protein concentration in the prime fluid, a shift in bound and unbound drug concentration may occur. Subsequent redistribution of drugs from the peripheral to the central compartments depends on protein binding, lipophilicity and $\mathrm{Vd}$.

(3) HT induces changes in metabolism and organ perfusion. Decreased hepatic flow causes subsequent changes in drug metabolism such as CYP2D6, which lead to impaired $\mathrm{CL}$ and drug accumulation with increased risk of toxicity [66]. This also potentially changes the child's response to the administered drug, thus the PD.

(4) SIRS occurs in one third of patients during the use of CPB. Main risk factors are the duration of CPB and the amount of fresh frozen plasma used. SIRS is associated with organ dysfunction and prolonged stay at the intensive care unit $[86,88]$. Inflammation and organ dysfunction reduce $C L$ of CYP3A-mediated drugs, such as midazolam [89]. Therefore children may be at risk of increased plasma concentrations and associated toxicity.

(5) Sequestration of drugs in the CPB system is a problem mainly for lipophilic drugs. The oxygenator has been identified as the main binding site of drugs. Coating of CPB tubing with heparin or biocompatible coating does not prevent absorption of drugs. Depending on the type of drug, up to $90 \%$ can be absorbed in the CPB circuit only minutes after administration [90,91].

Based on what has been discussed until now, it seems essential to explore the PK and PD related differences in these specific subpopulations, and population PK modeling or PK/ PD modeling is likely the ideal tool.

\subsection{Tolerance, drug dependency, and withdrawal}

All three patient groups described above are at risk for tolerance, drug dependency, and withdrawal after prolonged use of analgesics and sedatives. Tolerance is the decreased effect of a drug after prolonged exposure [92]. Several subcategories are described, such as innate (genetic) and PK tolerance. However, PD tolerance is the most appropriate definition since it refers to alterations at the distal end of the receptor [93]. The primary mechanisms of tolerance are receptor desensitization and upregulation of the CAMP pathway by various causes [92]. Different types of opioids have different effects on these mechanisms, leading to a variable potential to cause tolerance. The risk of developing tolerance is higher with prolonged duration of therapy, early developmental stage of the patient, male gender and the use of synthetic or shortacting opioids [92]. Tolerance and withdrawal symptoms can occur after five days of continuous infusion of opioids or benzodiazepines. Validated PD tools to assess withdrawal are the Withdrawal Assessment Tool-1 (WAT-1) and the Sophia Observation withdrawal Symptoms score (SOS) [34,45]. 
The clinical management of opioid tolerance and subsequent withdrawal is multimodal. Gradual weaning of opioids and replacing intravenous short-acting opioids with non-IV administered long-acting opioids is the recommended pharmacological therapy. Tolerance and withdrawal may be prevented or delayed by using daily interruption of sedation, nurse-controlled analgesia, or sequential rotation of analgesics $[45,92]$. Vet et al. showed that daily interruption of sedation in critically ill children decreased withdrawal symptoms, but did not improve clinical outcome [94]. The influence of PG on the cellular changes that lead to tolerance is not yet clear [92].

\section{Toward model-based dosing regimens using population PK modeling}

\subsection{Population PK modeling}

Population PK modeling paves the way to estimate PK parameters such as $\mathrm{CL}$ and $\mathrm{Vd}$ (based on concentration-time profiles) and to translate them in model-based dosing regimens, which should take into account information on (before, during and after) ECMO, cooling and CPB. The development of such dosing regimens using PK data is illustrated in Figure 2 and consists of a multi-step approach [95]:

(1) Optimal study design (based on preliminary data)

(2) Development and internal validation of PK model

(3) External validation of PK model

(4) Prospective validation in clinical study

(5) Proposed individualized dosing regimen

In the first step, previous PK studies must be evaluated on quality and the amount of data, such as clinical characteristics, drug concentrations in plasma, number of patients and time of sampling, retrieved from these studies [96]. In addition, attention should also be given to the abovementioned clinical characteristics when designing a new study. In the second step, a population PK model can be generated by using concentrations collected during studies or clinical practice. The development of a population PK-model is in three different steps: identification of the (a) structural model; (b) statistical model; (c) covariate model [97]. In the structural model, a population concentration-time curve is derived from the estimated parameters (Figure $1(\mathrm{~b})$ ) by pooling all concentrations obtained from all patients (Figure $1(a, b)$ ), whereas the statistical model quantifies all levels of variability (inter- individual variability (between-subjects), intra individual variability (within subject) and residual variability (assay error, error in recording sampling time, error in dosage preparation (Figure 2). Thus, the model takes into account the differences among observations (Figure 1(c)). In the covariate model, the use of patient and treatment characteristics is aimed is to (partly) understand and explain the inter-individual and intra-individual variability in the model parameters. These characteristics are often subdivided in maturational (age, weight), demographic (sex, race, genotype) and treatment characteristics (HT, ECMO, $\mathrm{CPB}$, surgery) but can also be time-varying covariates (for example pre- vs. post-CPB) (Figure 1). Different methods are available to quantify the influence of maturational changes on PK parameters of drugs in children, including allometric scaling using bodyweight-based functions with fixed exponents [98]. This can be combined with additional age-based maturation functions [99] or more datadriven approaches in which a systematic covariate analysis drives the definition of the statistically most appropriate relationship to describe maturational changes in observed data [97]. For morphine, the maturation of clearance has for instance been described using different parameterizations, based on bodyweight, age or a combination of both $[19,100-102]$. PK covariates can then be used to determine if and how dosing can be individualized to yield similar drug exposure for different subpopulations or even individual patients. This is irrespective of whether the source of inter-individual differences is maturational or nonmaturational and irrespective of the chosen parameterization for the influence of maturational changes on PK parameters, as long as the obtained relationships are properly validated $[37,103]$. For example, if a covariate, such as age, influences $\mathrm{Vd}$, the loading dose needs to be adjusted based on age. Covariates can thus be used to determine if and how dosing can be individualized [37].

The advantage of the population approach is that an entire concentration-time curve does not need to be obtained for every patient. This enables sparse blood sampling, which is preferred for neonates and children. Another advantage is that samples taken from clinical care or even scavenged sampling can be used, instead of designing complete experimental studies. The last part of the second step is the internal validation, in which the stability of the PK parameter estimates and the final model robustness are assessed to estimate the predictive property in terms of general trends in the population and on variability.

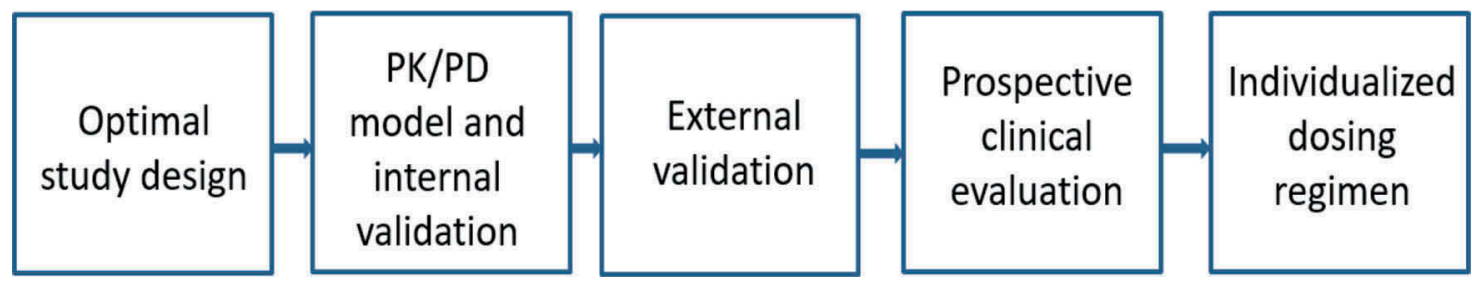

Figure 2. Multi-step approach for development of dosing regimens using PK/PD data.

Figure adapted with permission from Ince et al. [95]. 
The third step is the external validation, which implies investigating the accuracy of the model in new patients from the same population that was used for model building. This step is often not performed, however, due to the lack of an external dataset even though historical or clinical data can be used for this purpose. Prospective validation in a trial is the fourth step. During this step, the model is clinically tested to check whether the dosing advice derived from this model will reach the desired target range, and to identify patients in which the dosing regimen does not provide the correct concentrations or effects and must therefore be further elaborated. These might for instance include postoperative cardiac patients who have different PK parameters than postoperative noncardiac patients [78]. In the fifth step, individualized dosing regimens are achieved, and preferably incorporated in guidelines and formularies for use in clinical care.

\subsection{Looking beyond morphine and acetaminophen for post-operative pain}

Of the drugs commonly used to treat postoperative pain, morphine and acetaminophen have been extensively evaluated [34]. Concerning morphine for post-operative pain, all five steps leading to PK-based individualized dosing regimens have been completed for neonates and children (range 0-3 years of age) [20]. For both morphine and paracetamol, the PK has been identified and dosing guidelines have been proposed for the entire pediatric age range, although the last step (prospective evaluation) still deserves more attention [33,104]. It should be realized, however, that the morphine and acetaminophen dosages are recommended for major non-cardiac surgery only [19]. For other drugs used in the postoperative setting such as remifentanil (post-operative pain after cardiac surgery), diclofenac [105,106], nalbuphine [107], and ketorolac [108], PK-parameters derived from population models are available for certain routes of administration and in various pediatric age-groups. For drugs such as ibuprofen, oxycodone or fentanyl, models have not yet been developed for neonates or children for postoperative pain treatment.

In all PK models developed for neonates, infants, and children, maturational covariates (weight and/or age) are used to (partly) explain inter-patient variability in PK parameters. However, besides these maturational covariates, non-maturational covariates could also partly explain inter-individual as well as intra-individual variability. Both maturational and nonmaturational covariates should be taken into account when developing a dosing regimen in the studied population. The pre- or post-CPB period are examples of a non-maturational time-varying covariate emerging in the population PK model developed for remifentanil in infants and children undergoing cardiac surgery with CPB [81]. Vd was increased during and post-CPB compared to the pre-CPB period. The authors concluded that following this increase, an extra bolus of $25 \mu \mathrm{g}$ must be administered at the start of CPB compared to the pre$\mathrm{CPB}$ period to obtain an optimal target concentration of $14 \mathrm{ng} / \mathrm{ml}$ during CPB and post-CPB [81].

\section{Conclusion}

PK studies that report on PK parameters and its covariates for morphine, fentanyl, and acetaminophen are available, but data in specific subpopulations (HT, ECMO, or cardiac patients) are limited. PK data are also lacking for other commonly used opioids (alfentanil, remifentanil, nalbuphine) and non-opioids (e.g. ketorolac) in critically ill neonates, young infants, and children. PK parameters accounting for maturational and non-maturational covariates are important to develop population PK-based dosing advices for the pediatric age group. Besides disease characteristics and treatment modalities, PG should also be considered as a potential relevant covariate, but the impact of this covariate may depend on both maturational and non-maturational covariates. Irrespective of the compound, PD is often not investigated as end point in combination with PK parameters. PK models should be combined with validated PD outcome data to establish optimal evidence-based dosing regimens for specific populations and age groups.

\section{Expert opinion}

PK-parameters derived from population PK-models provide a first step toward individualized pain treatment in children. Besides maturational changes, non-maturational changes should be considered. In this review, this has been stressed for children undergoing $\mathrm{HT}$, on ECMO, or cardiac surgery, including CPB. PK parameters for these subpopulations are considered to differ from those for other pediatric populations in an ICU setting. While PK-based dosing advices are certainly useful, they will be clinically relevant only if prospectively evaluated in proof of principle studies in which both the PK and PD are considered. In this section, we will mainly focus on missing PK/PD data of routinely used analgesics and possibilities to turn this into personalized model-based dosing.

Most population PK-models report on maturational covariates (weight, age), mainly with respect to $C L$. The impact of non-maturational covariates $(E C M O, C P B$, disease state, inflammation) strongly depends on the type of patient group but can also vary within one and the same patient with time and disease progression. Given these differences in maturational and non-maturational covariates, attention should not only be paid to age and weight, but also to diagnosis and treatment modalities when developing PK-based dosing recommendations. For example, in critically ill children on ECMO, variation in PK parameters is extensive, with an increase in $\mathrm{Vd}$ between $5 \%$ and $400 \%$ for most drugs, and a decreased CL between $0 \%$ and $50 \%$ compared with children without ECMO [69]. In this respect, physiologically based (PB)-PK models can be considered to estimate the impact of non-maturational covariates on the disposition of specific compounds. PB-PK models use, besides PK information, also physiological information such as renal blood flow. These PB-PK models could be of interest, especially to explore whether results from one drug can be used to predict the PK for similar drugs; for example for drugs which are metabolized by the same route. An example is midazolam, which is mostly metabolized by CYP3A4. The PK parameters obtained from a PB-PK model of this drug could 
be evaluated for predictions for other drugs which are also CYP3A4 substrates. Physiological based models may be the future when dealing with large variability in maturational and non-maturational covariates. However, this will necessitate collecting accurate data on the (patho)physiology in these cases, including but not limited to, trends in caloric needs and metabolism, renal and hepatic blood flow and function or body composition, including fluid shifts. Development of accurate biomarkers to predict disease state, real time organ function, body composition and fluid shifts is essential in furthering our efforts to individualize and optimize drug dosing in these patients. Until such data become available, semiphysiological function approaches can be considered, as have been used for e.g. primary glomerular filtration rate driven aminoglycosides CL throughout pediatric life [109].

Another approach to investigate PK in critically ill children is micro-dosing. Its feasibility in children has been demonstrated by Mooij et al. [110]. Using a micro dose of $C^{14}$ labeled acetaminophen, maturational changes -in drug metabolism were explored without additional risk and only limited burden to the critically ill child [111]. As the feasibility of micro-dosing has been established, a similar approach can be considered in specific subpopulations like HT, ECMO or after cardiac surgery cases to quantify the impact of non-maturational covariates on drug disposition.

At present, PK parameters are not yet known for all routinely used analgesic drugs in children post-operatively or on ECMO. Regarding opioids, morphine and remifentanil have been well investigated in post-operative children $[19,20]$. Sufentanil and fentanyl are also often used after surgery, but the only report on sufentanil PK dates from 1987 [112]. The reported changes in PK of sufentanil in infants and children undergoing cardiac surgery may no longer be applicable in current clinical practice due to changes in CPB systems and perioperative and postoperative care. No report could be retrieved for fentanyl PK after cardiac surgery in children. Considering the clinical use of both fentanyl and sufentanil in post-operative patients, we would urge to investigate PK parameters with validated PD assessment, applying population PK/PD modeling techniques. Regarding non-opioids, acetaminophen has been investigated in post-operative infants, although the derived models have not yet been implemented in clinical practice $[33,55]$. Acetaminophen has been less well investigated in children after cardiac surgery. Considering the equipotency of IV acetaminophen to morphine observed in non-cardiac patients [55], with the benefit of less adverse drug reactions, this is certainly worth investigating. We consequently suggest to use the earlier mentioned methods (population PK, including semi-physiologic functions or PB-PK, micro-dosing) when focusing on these compounds because of the clinical need and the feasibility. In contrast, NSAIDs PK have not been investigated in children on ECMO. NSAIDs carry the risk of prolonged bleeding time and renal impairment, making physicians less inclined to use NSAIDs in these patients.

Once PK-models have been developed, PK/PD can be further investigated. The potential relevance of this PK/PD approach has recently been highlighted for morphine in ventilated preterm neonates undergoing endotracheal suctioning [113]. Using the item response theory, an intra-individual relationship between morphine concentrations and pain reduction in preterm neonates was unveiled. While the effect of morphine was small, this report illustrates the strengths of a PK/PD analysis approach. For morphine, we can conclude that PK-models have been developed and validated and that individual dosing regimens have been derived for postoperative pain $[19,20]$. However, the number of PD-models is still small, so further research should focus on the PD across the pediatric age range; which could probably help explaining the remaining variability [20]. One of the difficulties in PD-modeling is the use of multi-item scales (e.g. COMFORT) as an outcome variable. Different analgesics and sedatives are often administered simultaneously or a loading dose is administered, making it hard to link a dosing regimen to a pre-defined clinical end point, as other drugs than the one under investigation or the loading dose influence the outcome as reflected by the COMFORT score. Moreover, muscular paralysis, or the intervention itself $(\mathrm{HT})$ may also affect the possibilities to evaluate the PD.

\section{Funding}

P. Pokorna was supported by the General University Hospital RV- project [64165/2012]and an unrestricted research grant of the Intensive Care department in the Erasmus MC-Sophia Children's Hospital. G.A. Zeilmaker was supported by the ZonMw project [80836009840108]. P. Mian was supported by the Sophia Stichting Wetenschappelijk Onderzoek [SSWO S1608]. K Allegaert was supported by the Fund for Scientific Research, Flanders [fundamental clinical investigatorship $1800214 N]$. The research activities were further facilitated by the agency for innovation by Science and Technology in Flanders (IWT) through the SAFEPEDRUG project [IWT/SBO 130033].

\section{Declaration of interest}

The authors have no relevant affiliations or financial involvement with any organization or entity with a financial interest in or financial conflict with the subject matter or materials discussed in the manuscript. This includes employment, consultancies, honoraria, stock ownership or options, expert testimony, grants or patents received or pending, or royalties. Peer reviewers on this manuscript have no relevant financial or other relationships to disclose.

\section{References}

Papers of special note have been highlighted as either of interest $(\bullet)$ or of considerable interest (••) to readers.

1. Anand KJ, Hickey PR. Pain and its effects in the human neonate and fetus. N Engl J Med. 1987;317:1321-1329.

-. Key paper on the use of post-operative analgesics in neonates and infants.

2. Weisman SJ, Bernstein B, Schechter NL. Consequences of inadequate analgesia during painful procedures in children. Arch Pediatr Adolesc Med. 1998;152:147-149.

3. Berde CB, Jaksic T, Lynn AM, et al. Anesthesia and analgesia during and after surgery in neonates. Clin Ther. 2005;27:900-921.

4. Page GG. Are there long-term consequences of pain in newborn or very young infants? J Perinat Educ. 2004;13:10-17.

5. Taddio A, Katz J, llersich AL, et al. Effect of neonatal circumcision on pain response during subsequent routine vaccination. Lancet. 1997;349:599-603. 
6. de Graaf J, van Lingen RA, Valkenburg AJ, et al. Does neonatal morphine use affect neuropsychological outcomes at 8 to 9 years of age? Pain. 2013;154:449-458.

7. de Graaf J, van Lingen RA, Simons SH, et al. Long-term effects of routine morphine infusion in mechanically ventilated neonates on children's functioning: five-year follow-up of a randomized controlled trial. Pain. 2011;152:1391-1397.

8. Scholten W. Developing 'fully evidenced' paediatric pain guidelines. Eur J Hosp Pharm. 2013;20:262-263.

9. www.who.int.: Geneva: WHO; 2012 cited 2017 Dec 12. Available from: http://apps.who.int/iris/bitstream/10665/44540/1/ 9789241548120_Guidelines.pdf.

10. Hall RW, Anand KJS. Pain management in newborns. Clin Perinatol. 2014;41:895-924.

11. Poh YN, Poh PF, Buang SN, et al. Sedation guidelines, protocols, and algorithms in PICUs: a systematic review. Pediatr Crit Care Med. 2014;15:885-892.

12. Jebaraj B, Maitra S, Baidya DK, et al. Intravenous paracetamol reduces postoperative opioid consumption after orthopedic surgery: a systematic review of clinical trials. Pain Res Treat. 2013;2013:402510.

13. van Dijk M, de Boer JB, Koot $H M$, et al. The reliability and validity of the COMFORT scale as a postoperative pain instrument in 0 to 3 year-old infants. Pain. 2000;84:367-377.

14. Boerlage AA, Ista E, Duivenvoorden HJ, et al. The COMFORT behaviour scale detects clinically meaningful effects of analgesic and sedative treatment. Eur J Pain. 2015;19:473-479.

15. Matic $M$, de Wildt SN, Elens $L$, et al. SLC22A1/OCT1 genotype affects O-desmethyltramadol exposure in newborn infants. Ther Drug Monit. 2016;38:487-492.

16. Bradford LD. CYP2D6 allele frequency in European Caucasians, Asians, Africans and their descendants. Pharmacogenomics. 2002;3:229-243.

17. Foulkes T, Wood JN. Mechanisms of cold pain. Channels (Austin). 2007;1:154-160.

18. Rutkove SB. Effects of temperature on neuromuscular electrophysiology. Muscle Nerve. 2001;24:867-882.

19. Wang C, Sadhavisvam S, Krekels EH, et al. Developmental changes in morphine clearance across the entire paediatric age range are best described by a bodyweight-dependent exponent model. Clin Drug Investig. 2013;33:523-534.

20. Krekels EH, Tibboel D, de Wildt SN, et al. Evidence-based morphine dosing for postoperative neonates and infants. Clin Pharmacokinet. 2014;53:553-563.

21. Anand KJ, Stevens BJ, McGrath P. Pain in neonates and infants. 3rd ed. New York, United States: Elsevier; 2007.

22. Griffin JP. The textbook of pharmaceutical medicine. Hoboken, New Jersey, United States: Wiley-Blackwell; 2009.

23. Yamamoto Y, Valitalo PA, Wong YC, et al. Prediction of human CNS pharmacokinetics using a physiologically-based pharmacokinetic modeling approach. Eur J Pharm Sci. 2018;112:168-179.

24. Lam J, Koren G. P-glycoprotein in the developing human brain: A review of the effects of ontogeny on the safety of opioids in neonates. Ther Drug Monit. 2014;36:699-705.

25. Fernandez E, Perez R, Hernandez A, et al. Factors and mechanisms for pharmacokinetic differences between pediatric population and adults. Pharmaceutics. 2011;3:53-72.

26. Allegaert K, Simons SHP, Tibboel D, et al. Non-maturational covariates for dynamic systems pharmacology models in neonates, infants, and children: filling the gaps beyond developmental pharmacology. Eur J Pharm Sci. 2017

27. Kearns GL, Abdel-Rahman SM, Alander SW, et al. Developmental pharmacology-drug disposition, action, and therapy in infants and children. N Engl J Med. 2003;349:1157-1167.

-. Key paper on developmental pharmacology in infants and children.

28. van Den Anker JN, Schwab M, Kearns GL. Developmental pharmacokinetics. Handb Exp Pharmacol. 2011;205:51-75.

29. Mooij MG, Schwarz UI, de Koning BA, et al. Ontogeny of human hepatic and intestinal transporter gene expression during childhood: age matters. Drug Metab Dispos. 2014;42:1268-1274.
30. Blake MJ, Castro L, Leeder JS, et al. Ontogeny of drug metabolizing enzymes in the neonate. Semin Fetal Neonatal Med. 2005;10:123-138.

31. Allegaert K. Tailored tools to improve pharmacotherapy in infants. Expert Opin Drug Metab Toxicol. 2014;10:1069-1078.

32. Krekels EH, Tibboel D, Knibbe CA. Pediatric pharmacology: current efforts and future goals to improve clinical practice. Expert Opin Drug Metab Toxicol. 2015;11:1679-1682.

33. Wang C, Allegaert K, Tibboel D, et al. Population pharmacokinetics of paracetamol across the human age-range from (pre)term neonates, infants, children to adults. J Clin Pharmacol. 2014;54:619-629.

34. Baarslag MA, Allegaert K, Knibbe CA, et al. Pharmacological sedation management in the paediatric intensive care unit. J Pharm Pharmacol. 2016;69:498-513.

35. Krekels EH, van Ham S, Allegaert K, et al. Developmental changes rather than repeated administration drive paracetamol glucuronidation in neonates and infants. Eur J Clin Pharmacol. 2015;71:1075-1082.

36. Vet $N$, de Hoog $M$, Tibboel $D$, et al. The effect of inflammation on drug metabolism: a focus on pediatrics. Drug Discov Today. 2011;16:435-442.

37. Anderson BJ, Allegaert K, Holford NH. Population clinical pharmacology of children: general principles. Eur J Pediatr. 2006;165:741-746.

38. Wildschut ED, de Wildt SN, Mathot RAA, et al. Effect of hypothermia and extracorporeal life support on drug disposition in neonates. Semin Fetal Neonatal Med. 2013;18:23-27.

\section{- Overview of mechanisms on drug deposition and ECMO.}

39. Zwiers AJ, de Wildt SN, Hop WC, et al. Acute kidney injury is a frequent complication in critically ill neonates receiving extracorporeal membrane oxygenation: a 14-year cohort study. Crit Care. 2013;17:R151.

40. Leeder JS, Kearns GL. Interpreting pharmacogenetic data in the developing neonate: the challenge of hitting a moving target. Clin Pharmacol Ther. 2012;92:434-436.

41. Allegaert K, Van Den Anker JN, De Hoon JN, et al. Covariates of tramadol disposition in the first months of life. $\mathrm{Br} J$ Anaesth. 2008;100:525-532.

42. Orliaguet G, Hamza J, Couloigner V, et al. A case of respiratory depression in a child with ultrarapid CYP2D6 metabolism after tramadol. Pediatrics. 2015;135:e7535.

43. Kelly LE, Rieder $M$, van den Anker J, et al. More codeine fatalities after tonsillectomy in North American children. Pediatrics. 2012;129:e13437.

44. Roka A, Melinda KT, Vasarhelyi B, et al. Elevated morphine concentrations in neonates treated with morphine and prolonged hypothermia for hypoxic ischemic encephalopathy. Pediatrics. 2008;121:e844-9.

45. Harris J, Ramelet AS, van Dijk M, et al. Clinical recommendations for pain, sedation, withdrawal and delirium assessment in critically ill infants and children: an ESPNIC position statement for healthcare professionals. Intensive Care Med. 2016;42:972-986.

46. Knosgaard KR, Foster DJ, Kreilgaard M, et al. Pharmacokinetic models of morphine and its metabolites in neonates: systematic comparisons of models from the literature, and development of a new meta-model. Eur J Pharm Sci. 2016;92:117-130.

47. Crellin DJ, Harrison D, Santamaria N, et al. Systematic review of the face, legs, activity, cry and consolability scale for assessing pain in infants and children: is it reliable, valid, and feasible for use? Pain. 2015;156:2132-2151.

48. Krechel SW, Bildner J. CRIES: a new neonatal postoperative pain measurement score. Initial testing of validity and reliability. Paediatr Anaesth. 1995:5:53-61.

49. Matic M, Norman E, Rane A, et al. Effect of UGT2B7900G $>A$ ( $-842 G>A$; rs7438135) on morphine glucuronidation in preterm newborns: results from a pilot cohort. Pharmacogenomics. 2014;15:1589-1597.

50. Matic $M$, Simons SH, van Lingen RA, et al. Rescue morphine in mechanically ventilated newborns associated with combined OPRM 1 and COMT genotype. Pharmacogenomics. 2014;15:1287-1295.

51. Meesters NJ, van Dijk M, Knibbe CA, et al. Infants operated on for necrotizing enterocolitis: towards evidence-based pain guidelines. Neonatology. 2016;110:190-197. 
52. Shay JE, Kattail D, Morad A, et al. The postoperative management of pain from intracranial surgery in pediatric neurosurgical patients. Paediatr Anaesth. 2014;24:724-733.

53. Anand KJS, Hickey PR. Halothane-morphine compared with highdose sufentanil for anesthesia and postoperative analgesia in neonatal cardiac surgery. New England J Med. 1992.

54. Chou R, Gordon DB, de Leon-Casasola OA, et al. Management of postoperative pain: a clinical practice guideline from the American Pain Society, the American Society of Regional Anesthesia and Pain Medicine, and the American Society of Anesthesiologists' Committee on Regional Anesthesia, Executive Committee, and Administrative Council. J Pain. 2016;17:131-157.

55. Ceelie I, de Wildt SN, van Dijk M, et al. Effect of intravenous paracetamol on postoperative morphine requirements in neonates and infants undergoing major noncardiac surgery a randomized controlled trial. Jama-Journal Am Med Assoc. 2013;309:149-154.

56. Anderson BJ, Pons G, Autret-Leca E, et al. Pediatric intravenous paracetamol (propacetamol) pharmacokinetics: a population analysis. Paediatr Anaesth. 2005;15:282-292.

57. Kokki H. Ketoprofen pharmacokinetics, efficacy, and tolerability in pediatric patients. Paediatr Drugs. 2010;12:313-329.

58. Rollason V, Desmeules JA. Use of metamizole in children and the risk of agranulocytosis: is the benefit worth the risk? Eur J Anaesthesiol. 2015;32:837-838.

59. Schnabel A, Reichl SU, Meyer-Friessem C, et al. Tramadol for postoperative pain treatment in children. Cochrane Database Syst Rev. 2015;CD009574.

60. Schnabel A, Reichl SU, Zahn PK, et al. Nalbuphine for postoperative pain treatment in children. Cochrane Database Syst Rev.. 2014; CD009583.

61. Baley K, Michalov K, Kossick MA, et al. Intravenous acetaminophen and intravenous ketorolac for management of pediatric surgical pain: a literature review. Aana J. 2014;82:53-64.

62. Monk JP, Beresford R, Ward A. Sufentanil. A review of its pharmacological properties and therapeutic use. Drugs. 1988;36:286-313.

63. Lundeberg S, Roelofse JA. Aspects of pharmacokinetics and pharmacodynamics of sufentanil in pediatric practice. Paediatr Anaesth. 2011;21:274-279.

64. Greeley WJ, de Bruijn NP. Changes in sufentanil pharmacokinetics within the neonatal period. Anesth Analg. 1988;67:86-90.

65. Pokorna P, Wildschut ED, Vobruba V, et al. The impact of hypothermia on the pharmacokinetics of drugs used in neonates and young infants. Curr Pharm Des. 2015;21:5705-5724.

66. van den Broek MP, Groenendaal F, Egberts AC, et al. Effects of hypothermia on pharmacokinetics and pharmacodynamics: a systematic review of preclinical and clinical studies. Clin Pharmacokinet. 2010;49:277-294.

67. Meurs KV, Lally KP, Peek G, et al. ECMO; extracorporeal cardiopulmonary support in critical care. 3rd ed. Extracorporeal Life Support Organization; 2005. Ann Arbor, MI.

68. Mulla H, Lawson $G$, Woodland ED, et al. Effects of neonatal extracorporeal membrane oxygenation circuits on drug disposition. Curr Ther Res Clin Exp. 2000;61:838-848.

69. Wildschut ED, van Saet A, Pokorna P, et al. The impact of extracorporeal life support and hypothermia on drug disposition in critically III infants and children. Pediatr Clin North Am. 2012;59:1184-1204.

70. Shekar K, Fraser JF, Smith MT, et al. Pharmacokinetic changes in patients receiving extracorporeal membrane oxygenation. J Crit Care. 2012;27:741.e9-.e18.

71. Dagan O, Klein J, Gruenwald C, et al. Preliminary studies of the effects of extracorporeal membrane-oxygenator on the disposition of common pediatric drugs. Ther Drug Monit. 1993;15:263-266.

72. Peters JW, Anderson BJ, Simons SH, et al. Morphine metabolite pharmacokinetics during venoarterial extra corporeal membrane oxygenation in neonates. Clin Pharmacokinet. 2006;45:705-714.

73. Association of Paediatric Anaesthetists of Great B, Ireland. Good practice in postoperative and procedural pain management, 2nd edition. Paediatr Anaesth. 2012;22(Suppl 1):1-79.

74. Lucas SS, Nasr VG, Ng AJ, et al. Pediatric cardiac intensive care society 2014 consensus statement: pharmacotherapies in cardiac critical care: sedation, analgesia and muscle relaxant. Pediatr Crit Care Med. 2016;17:S3-S15.

75. Dagan O, Klein J, Bohn D, et al. Morphine pharmacokinetics in children following cardiac surgery: effects of disease and inotropic support. J Cardiothorac Vasc Anesth. 1993;7:396-398.

76. Lynn A, Nespeca MK, Bratton SL, et al. Clearance of morphine in postoperative infants during intravenous infusion: the influence of age and surgery. Anesth Analg. 1998;86:958-963.

- Overview of post-operative morphine consumption leading to dose reccomendations.

77. Elkomy MH, Drover DR, Glotzbach KL, et al. Pharmacokinetics of morphine and its metabolites in infants and young children after congenital heart surgery. Aaps J. 2016;18:124-133.

78. van Saet A, de Wildt SN, Knibbe CA, et al. The effect of adult and pediatric cardiopulmonary bypass on pharmacokinetic and pharmacodynamic parameters. Curr Clin Pharmacol. 2013;8:297-318.

- Review describing influence of CPB on PK and PD in neonates and children.

79. Valkenburg AJ, Calvier EA, van Dijk M, et al. Pharmacodynamics and pharmacokinetics of morphine after cardiac surgery in children with and without down syndrome. Pediatr Crit Care Med. 2016;17:930-938.

80. Rigby-Jones AE, Priston MJ, Sneyd JR, et al. Remifentanil-midazolam sedation for paediatric patients receiving mechanical ventilation after cardiac surgery. Br J Anaesth. 2007;99:252-261.

81. Sam WJ, Hammer GB, Drover DR. Population pharmacokinetics of remifentanil in infants and children undergoing cardiac surgery. BMC Anesthesiol. 2009;9.

82. Kleiber N, de Wildt SN, Cortina G, et al. Clonidine as a first-line sedative agent after neonatal cardiac surgery: retrospective cohort study. Pediatr Crit Care Med. 2016;17:332-341.

83. Wolf A, McKay A, Spowart C, et al. Prospective multicentre randomised, double-blind, equivalence study comparing clonidine and midazolam as intravenous sedative agents in critically ill children: the SLEEPS (Safety profiLe, Efficacy and Equivalence in Paediatric intensive care Sedation) study. Health Technol Assess. 2014;18:1-212.

84. Potts $A L$, Larsson $P$, Eksborg $S$, et al. Clonidine disposition in children; a population analysis. Paediatr Anaesth. 2007;17:924-933.

85. Su F, Gastonguay MR, Nicolson SC, et al. Dexmedetomidine pharmacology in neonates and infants after open heart surgery. Anesth Analg. 2016;122:1556-1566.

86. Hall Rl. Cardiopulmonary bypass and the systemic inflammatory response: effects on drug action. J Cardiothorac Vasc Anesth. 2002;16:83-98.

87. Ji B, Undar A. An evaluation of the benefits of pulsatile versus nonpulsatile perfusion during cardiopulmonary bypass procedures in pediatric and adult cardiac patients. Asaio J. 2006;52:357-361.

88. Boehne $M$, Sasse $M$, Karch $A$, et al. Systemic inflammatory response syndrome after pediatric congenital heart surgery: incidence, risk factors, and clinical outcome. J Card Surg. 2017;32:116-125.

89. Vet NJ, Brussee JM, de Hoog M, et al. Inflammation and organ failure severely affect midazolam clearance in critically ill children. Am J Respir Crit Care Med. 2016.

90. Hammaren E, Rosenberg PH, Hynynen M. Coating of extracorporeal circuit with heparin does not prevent sequestration of propofol in vitro. Br J Anaesth. 1999;82:38-40.

91. Koren G, Crean P, Klein J. Sequestration of fentanyl by the cardiopulmonary bypass (CPBP). Eur J Clin Pharmacol. 1984;27:51-56.

92. Anand KJ, Willson DF, Berger J, et al. Tolerance and withdrawal from prolonged opioid use in critically ill children. Pediatrics. 2010;125:e1208-25.

- Review on tolerance and withdrawal from opioid use in critically ill children.

93. Tobias JD. Tolerance, withdrawal, and physical dependency after long-term sedation and analgesia of children in the pediatric intensive care unit. Crit Care Med. 2000;28:2122-2132. 
94. Vet NJ, de Wildt SN, Verlaat CW, et al. A randomized controlled trial of daily sedation interruption in critically ill children. Intensive Care Med. 2016;42:233-244.

95. Ince I, de Wildt SN, Tibboel D, et al. Tailor-made drug treatment for children: Creation of an infrastructure for data-sharing and population PK-PD modeling. Drug Discov Today. 2009;14:316-320.

96. Aarons L, Ogungbenro K. Optimal design of pharmacokinetic studies. Basic Clin Pharmacol Toxicol. 2010;106:250-255.

97. de Cock RF, Piana C, Krekels EH, et al. The role of population PK-PD modelling in paediatric clinical research. Eur J Clin Pharmacol. 2011;67(Suppl 1):5-16.

- Underscribing the role and feasibility of PK-PD modelling in pediatric clinical research.

98. Mahmood I. Prediction of drug clearance in premature and mature neonates, infants, and children $</=2$ years of age: a comparison of the predictive performance of 4 allometric models. J Clin Pharmacol. 2016;56:733-739.

99. Anderson BJ, Holford NH. Mechanistic basis of using body size and maturation to predict clearance in humans. Drug Metab Pharmacokinet. 2009;24:25-36.

100. Bouwmeester NJ, Anderson BJ, Tibboel D, et al. Developmental pharmacokinetics of morphine and its metabolites in neonates, infants and young children. Br J Anaesth. 2004;92:208-217.

101. Anand KJ, Anderson BJ, Holford NH, et al. Morphine pharmacokinetics and pharmacodynamics in preterm and term neonates: secondary results from the NEOPAIN trial. $\mathrm{Br} J$ Anaesth. 2008;101:680-689.

102. Knibbe CA, Krekels EH, van den Anker JN, et al. Morphine glucuronidation in preterm neonates, infants and children younger than 3 years. Clin Pharmacokinet. 2009;48:371-385.

103. Krekels EH, van Hasselt JG, Tibboel D, et al. Systematic evaluation of the descriptive and predictive performance of paediatric morphine population models. Pharm Res. 2011;28:797-811.
104. Mian PKC, Tibboel D, Allegaert K. What dose of paracetamol is needed in neonates with pain? Arch of Dis in Childh. 2017. Forthcoming.

105. Standing JF, Howard RF, Johnson A, et al. Population pharmacokinetics of oral diclofenac for acute pain in children. $\mathrm{Br} J$ Clin Pharmacol. 2008;66:846-853.

106. van der Marel CD, Anderson BJ, Romsing J, et al. Diclofenac and metabolite pharmacokinetics in children. Paediatr Anaesth. 2004;14:443-451.

107. Bressolle F, Khier S, Rochette A, et al. Population pharmacokinetics of nalbuphine after surgery in children. Br J Anaesth. 2011;106:558-565.

108. Mohammed BS, Engelhardt T, Hawwa AF, et al. The enantioselective population pharmacokinetics of intravenous ketorolac in children using a stereoselective assay suitable for microanalysis. J Pharm Pharmacol. 2015;67:1179-1187.

109. de Cock RF, Allegaert K, Brussee JM, et al. Simultaneous pharmacokinetic modeling of gentamicin, tobramycin and vancomycin clearance from neonates to adults: towards a semi-physiological function for maturation in glomerular filtration. Pharm Res. 2014;31:2643-2654.

110. Mooij MG, de Koning BA, Huijsman ML, et al. Ontogeny of oral drug absorption processes in children. Expert Opin Drug Metab Toxicol. 2012;8:1293-1303.

111. Mooij MG, van Duijn E, Knibbe CA, et al. Successful use of [14C] paracetamol microdosing to elucidate developmental changes in drug metabolism. Clin Pharmacokinet. 2017.

112. Davis PJ, Cook DR, Stiller RL, et al. Pharmacodynamics and pharmacokinetics of high-dose sufentanil in infants and children undergoing cardiac surgery. Anesth Analg. 1987;66:203208.

113. Valitalo PA, van Dijk $M$, Krekels EH, et al. Pain and distress caused by endotracheal suctioning in neonates is better quantified by behavioural than physiological items: a comparison based on item response theory modelling. Pain. 2016;157:1611-1617. 